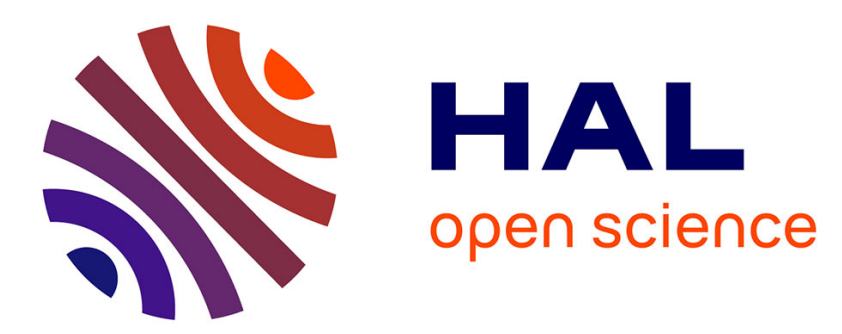

\title{
Étude et réalisation d'un spectrographe sous vide destiné a l'étude de l'émission dans l'U.V. lointain d'un jet de plasma en atmosphère raréfiée
}

Guy Bastian, Jean-Louis Queffelec

\section{- To cite this version:}

Guy Bastian, Jean-Louis Queffelec. Étude et réalisation d'un spectrographe sous vide destiné a l'étude de l'émission dans l'U.V. lointain d'un jet de plasma en atmosphère raréfiée. Revue de Physique Appliquée, 1968, 3 (3), pp.243-249. 10.1051/rphysap:0196800303024300 . jpa-00242855

\section{HAL Id: jpa-00242855 https://hal.science/jpa-00242855}

Submitted on 1 Jan 1968

HAL is a multi-disciplinary open access archive for the deposit and dissemination of scientific research documents, whether they are published or not. The documents may come from teaching and research institutions in France or abroad, or from public or private research centers.
L'archive ouverte pluridisciplinaire HAL, est destinée au dépôt et à la diffusion de documents scientifiques de niveau recherche, publiés ou non, émanant des établissements d'enseignement et de recherche français ou étrangers, des laboratoires publics ou privés. 


\title{
ÉTUDE ET RÉALISATION D'UN SPEGTROGRAPHE SOUS VIDE DESTINÉ A L'ÉTUDE DE L'ÉMISSION DANS L'U.V. LOINTAIN D’UN JET DE PLASMA EN ATMOSPHÈRE RARÉFIÉE (1)
}

\author{
Par Guy BASTIAN et JeAn-Louis QUEFFELEG, \\ Laboratoire de Physique Moléculaire, Faculté des Sciences, Rennes.
}

(Reçu le 21 mars 1968.)

\begin{abstract}
Résumé. - Nous avons réalisé et mis au point un spectrographe à réseau sous vide, pour le domaine des longueurs d'ondes comprises entre 900 et $2500 \AA$. Ce spectrographe est muni d'une source pour U.V. lointain intense que nous avons également mise au point et qui est constituée par un jet de plasma de gaz rare dans une enceinte où la pression est réglable de quelques torrs à 760 torrs.
\end{abstract}

Abstract. - A vacuum grating spectrograph for the 900-2 $500 \AA$ wavelength region has been constructed and adjusted. A high intensity vacuum U.V. source is used with this spectrograph. This source consists of a rare-gas plasma jet in a chamber where the pressure is adjustable from a few torrs to 760 torrs.

1. Introduction. - Divers travaux déjà réalisés au laboratoire par Queffelec [1], Velly [2] et Le Men [3] ont permis l'étude de plusieurs modèles d'arcs stabilisés et de chalumeaux à plasma fonctionnant à la pression atmosphérique. Des mesures spectroscopiques dans le visible, au moyen d'un spectrographe Jarrel Ash (dispersion : 2,55 $\AA / \mathrm{mm}$ dans le 1 er ordre), ont conduit notamment à la mesure, par diverses méthodes, de la distribution radiale des températures d'un plasma à symétrie cylindrique. Par le présent travail, nous étendons le domaine d'étude à l'ultra-violet lointain par la production de plasma en atmosphère raréfiée.

De nombreux auteurs ont construit des spectrographes pour l'U.V. lointain; citons entre autres Romand et Vodar [4], [5], Isaacs, Price et Ridley [6], Dirtchburn [7], Milazzò [8] et Burlitch et Duke [9]. Nous préciserons dans les $\S \S 2$ et 3 les caractéristiques du spectrographe adopté et décrirons la source réalisée $(\S 4)$ : un plasmatron sous atmosphère raréfiée.

2. Etude théorique du spectrographe. - Nous nous sommes proposés de réaliser un montage simple permettant l'étude d'une région spectrale précise $(900$ à $2500 \AA$ ).

Dans l'U.V. lointain, le seul matériel de spectroscopie acceptable est le réseau sphérique concave de rayon de courbure $R$ qui focalise directement la lumière sans l'intermédiaire de lentilles ou de miroirs supplémentaires qui provoqueraient des pertes énormes d'intensité lumineuse. Définissant le cercle de

(1) Ce travail a été financé par la D.R.M.E.
Rowland comme tangent au réseau en son centre et de diamètre égal au rayon de courbure $R$ du réseau, on démontre que la lumière issue d'une fente située sur le cercle et normale à son plan donne, après analyse par le réseau, des raies spectrales parallèles à la fente et passant par ce cercle (fig. 1). Des trois montages possibles : de Rowland, d'Eagle et de Paschen-Runge, nous avons choisi celui de PaschenRunge. Avec ce montage (fig. 1), en se limitant à un certain domaine spectral, il est possible de maintenir en position fixe fente, réseau et film récepteur.

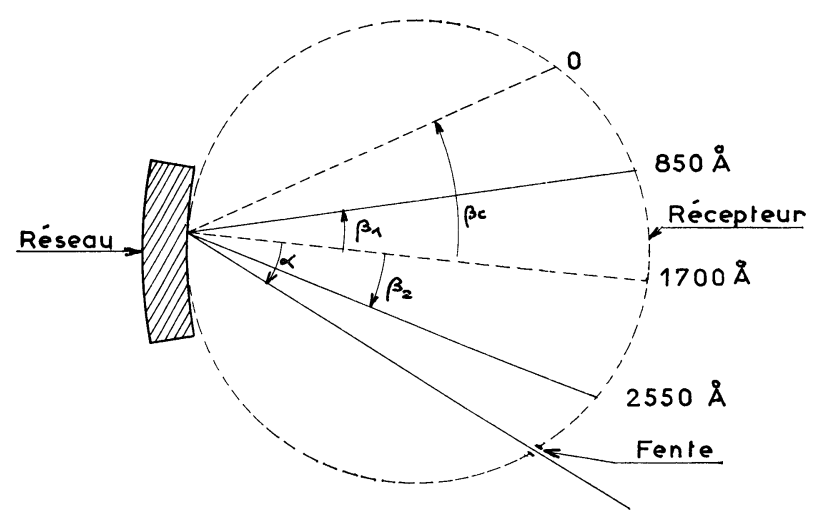

Fig. 1. - Montage de Paschen-Runge.

Nous avons utilisé un réseau concave (Bausch et Lomb) de rayon de courbure $R=998,8 \mathrm{~mm}$, à 1200 traits par $\mathrm{mm}$. Ce réseau est « blazé 》 à $\lambda_{\mathrm{B}}=1700 \AA$ et l'angle de «blaze » est $\theta=5^{\circ} 52^{\prime}$. 
Sawyer [10] indique que :

1) Lorsque le réseau est plan, la région spectrale " blazée " s'étend approximativement de $\lambda_{B} / 2$ $(=850 \AA)$ à $3 \lambda_{\mathrm{B}} / 2 \quad(=2550 \AA)$ dans le premier ordre, et que :

2) Pour un réseau concave, la région «blazée " est encore plus étendue. Toutefois, nous nous sommes limités (au moins en ce qui concerne la borne supérieure) à l'intervalle précédent.

D'autre part, les angles d'incidence $(\alpha)$ et d'observation $(\beta)$ étant faibles, ce réseau donne une dispersion sensiblement constante de $8,3 \AA / \mathrm{mm}$ dans le $11^{\text {er }}$ ordre.

La valeur de l'angle d'incidence a été fixée à $\alpha=11^{\circ} 44^{\prime}$. Dans ces conditions, $\beta_{1}=-5^{\circ} 52^{\prime}$ pour $\lambda_{1}=850 \AA$ et $\beta_{2}=5^{\circ} 52^{\prime}$ pour $\lambda_{2}=2550 \AA$. La tache centrale $\lambda=0$ est obtenue pour $\beta_{\mathrm{c}}=-11^{\circ} 44^{\prime}$ (fig. 1).

La théorie du réseau concave a été établie par Beutler [11]. Elle nous permet de prévoir les aberrations et les défauts des spectres dans notre domaine d'étude, d'autant mieux que le réseau considéré par cet auteur a le même nombre de traits par millimètre et même rayon de courbure que le nôtre. Des représentations graphiques de ces aberrations sont fournies en fonction des paramètres $\alpha$ et $\beta$.

Étant donné la valeur de $\alpha$ et les limites entre lesquelles varie $\beta$, on a déterminé que l'astigmatisme (mesuré par la hauteur du spectre quand la fente est réduite à un point) est compris entre 0,8 et $1,2 \mathrm{~mm}$. L'astigmatisme est donc très faible.

Il est intéressant de déterminer la position devant la fente d'une droite lumineuse perpendiculaire à celle-ci et dont le système fente-réseau donne une image ponctuelle sur le cercle de Rowland. Une lecture d'abaque indique que cette droite se trouve à environ $0,05 R$, soit, pour $R=100 \mathrm{~cm}$, à $5 \mathrm{~cm}$ en avant de la fente pour le domaine spectral étudié.

Un cylindre de plasma ayant cette droite pour axe donnera un spectre tel qu'une portion de raie spectrale corresponde " biunivoquement » à une tranche de plasma (fig. 2) (Griem [12]).

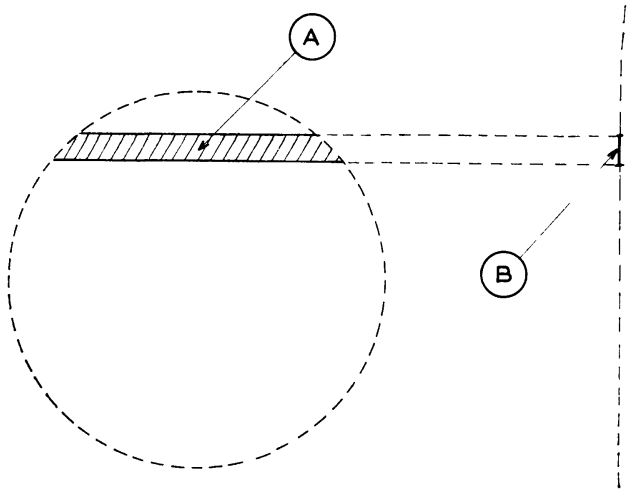

FIG. 2.

A, Portion de plasma ; B, Portion de raie correspondante

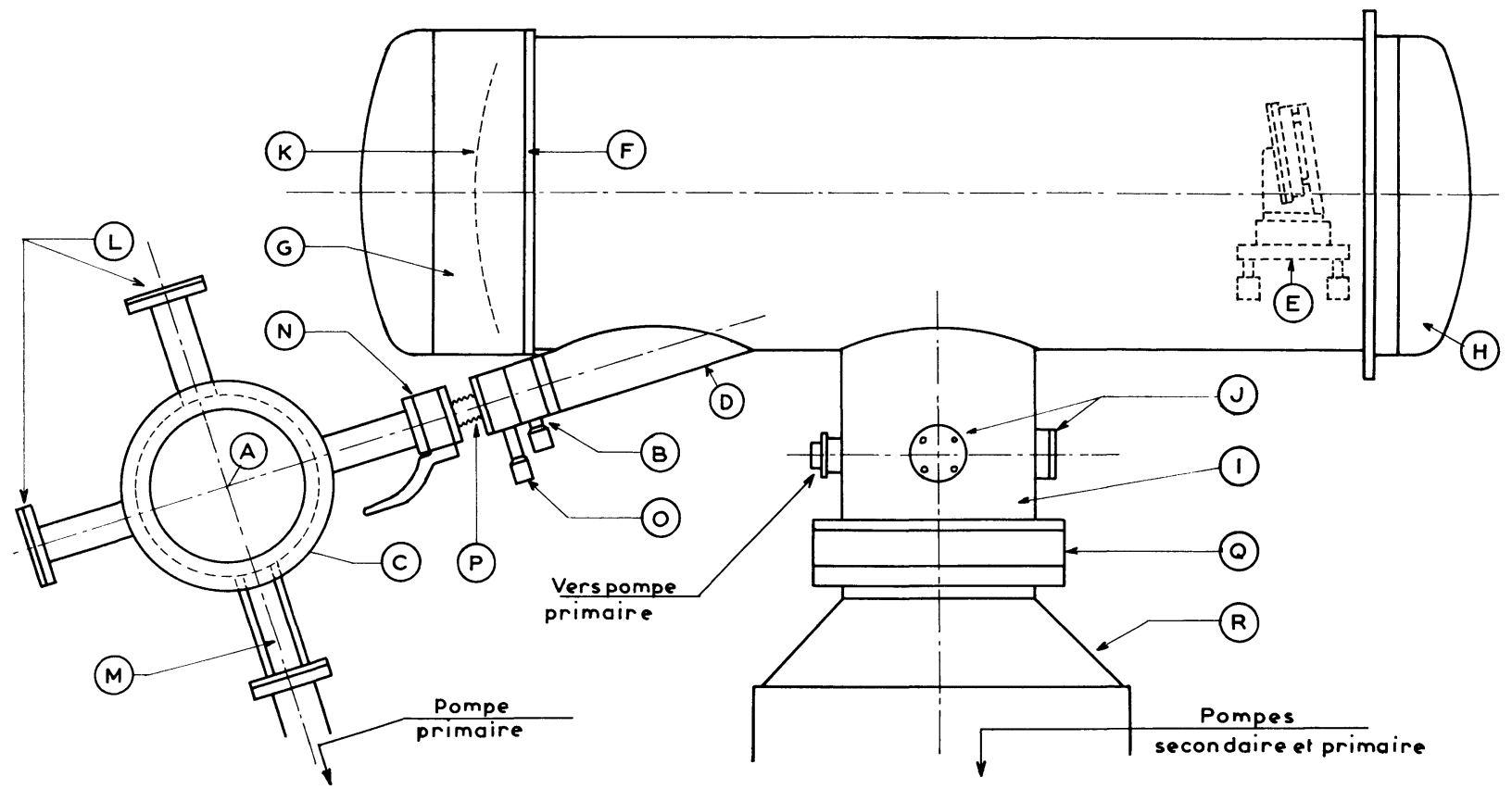

Fig. 3. - Schéma général du montage : A, Axe du générateur de plasma; $B$, Fente d'entrée ; C, Enceinte du plasmatron; D, Conduit oblique; $\mathrm{E}$, Support du réseau; F, Bride pleine; G, Porte articulée ; $H$, Bride arrière; I, Piquage vertical; J, Piquages pour jauges à vide; K, emplacement du film suivant le cercle de Rowland; L, Piquages d'observation; M, Piquage pour jauge à vide ; N, Vanne ; O, Réglage hauteur de fente ; $\mathrm{P}$, Raccord souple ; Q, Vanne papillon; $\mathrm{R}$, Piège à air liquide. 
Dans notre montage, il n'est pas possible de disposer le jet de plasma à $5 \mathrm{~cm}$ de la fente du spectrographe, mais tout au moins, pour les longueurs d'onde supérieures à $1100 \AA$, on pourra former l'image du dard à l'emplacement désiré au moyen d'une lentille en fluorure de lithium ou d'un miroir concave aluminié.

La hauteur de la fente est limitée à 2 ou $3 \mathrm{~mm}$; lorsque les angles $\alpha$ et $\beta$ sont petits, comme c'est le cas dans notre montage, l'aberration de coma n'est pas gênante. C'est effectivement ce que l'on observe et il n'y a pas lieu de diminuer la hauteur utile des traits du réseau.

Il existe enfin une distorsion des raies spectrales due à la courbure d'astigmatisme et à la courbure d'enveloppe; étant donné la faible hauteur des spectres, on aboutit au maximum à une courbure correspondant à une flèche de l'ordre de $0,5 \times 10^{-2} \mathrm{~mm}$.

3. Réalisation pratique du spectrographe. - L'axe A du générateur de plasma ou plasmatron est disposé horizontalement (fig. 3). La fente d'entrée (3 B) du spectrographe est disposée parallèlement à l'axe du dard (toutefois, il est également possible de la placer perpendiculairement à cet axe). La fente d'entrée est horizontale et le plan du cercle de Rowland vertical. Le plasmatron est adapté hermétiquement à une enceinte cylindrique coaxiale (3 C) dans laquelle règne une pression réduite ( 1 à 760 torrs). Cette cuve est raccordée de façon étanche à celle du spectrographe. L'ensemble du montage, réalisé d'après nos plans, en acier inoxydable, est posé sur un bâti en tube d'acier.

La cuve du spectrographe est un tube cylindrique où le rayonnement étudié pénètre par un conduit oblique $(3 \mathrm{D})$ situé au-dessous, incliné à $17^{\circ} 45^{\prime}\left( \pm 15^{\prime}\right)$ et auquel s'adapte la fente d'entrée $(3 \mathrm{~B})$.

Dans cette cuve, et en retrait de la bride arrière $(3 \mathrm{H})$, se place le réseau dans son support $(3 \mathrm{E})$ (fig. 4). La bride pleine ( $3 \mathrm{~F}$ ) fermant la face avant est pourvue d'une fenêtre verticale, passant par l'axe de la cuve, et d'où sort la lumière formant le spectre.

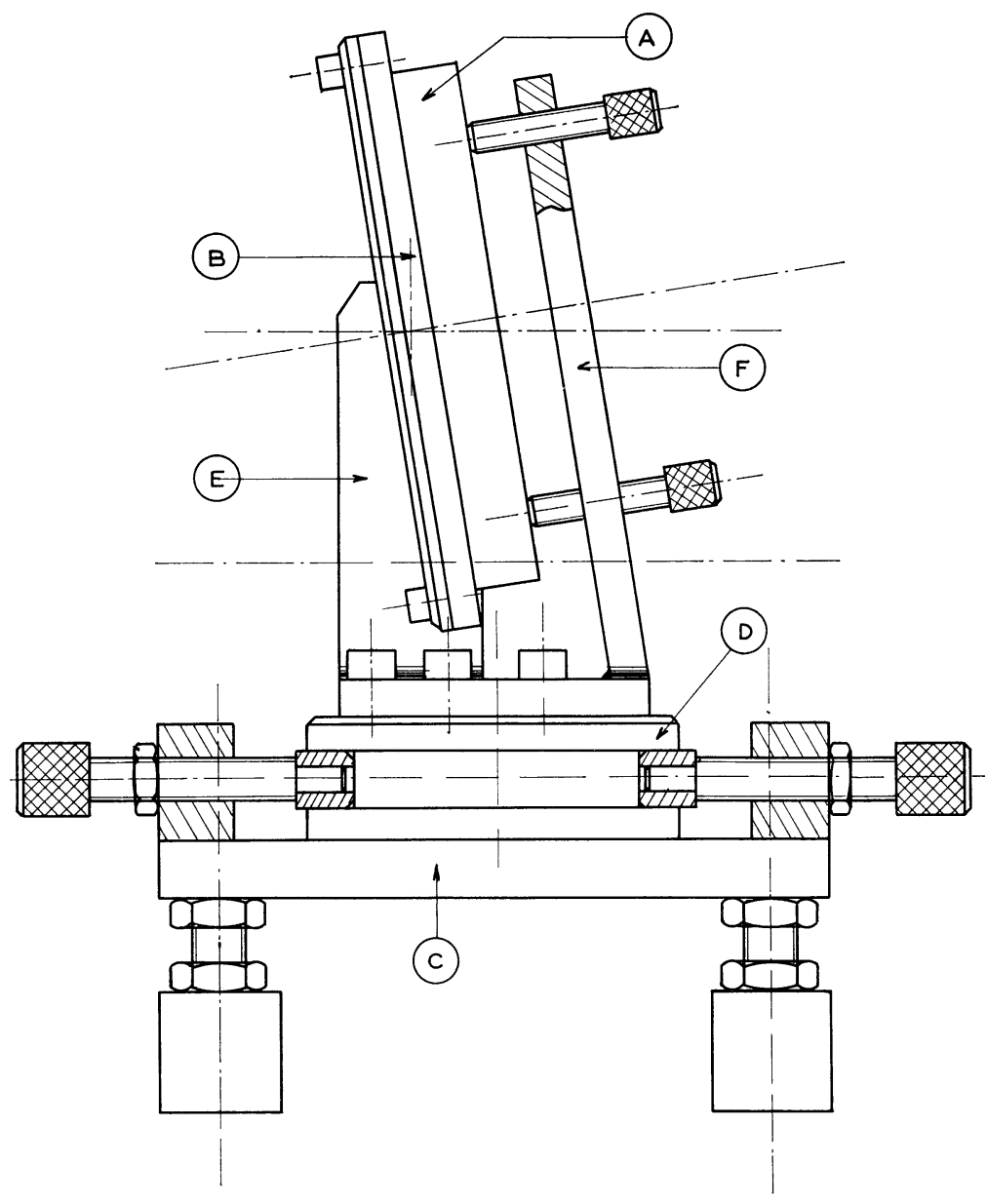

FrG. 4. - Support du réseau :

A, Boîte cylindrique ; B, Tourillon ; C, Première plate-forme ; D, Deuxième plate-forme ; E et F, Supports. 


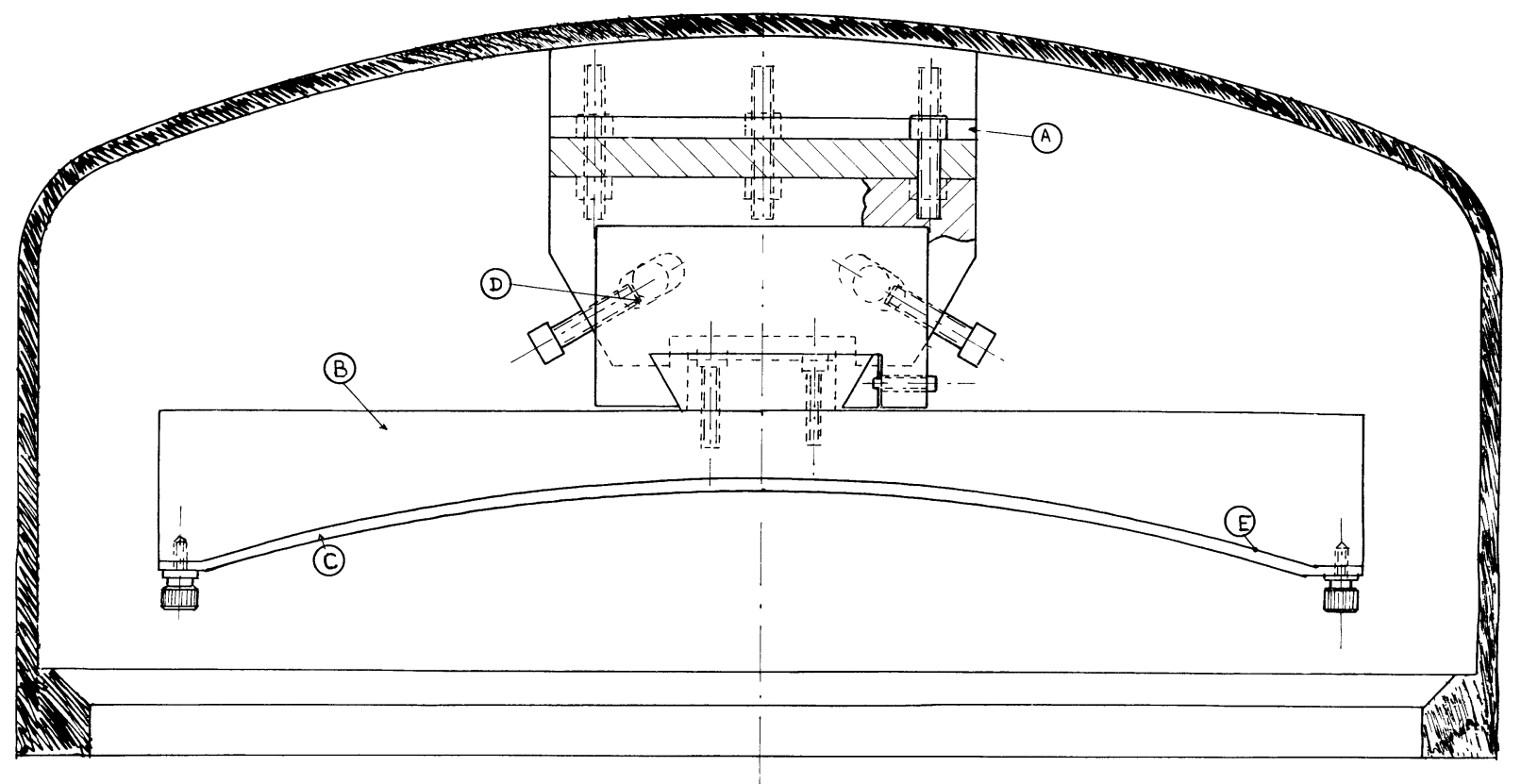

FIG. 5. - Châssis porte-film :

A, Support fixe; B, Châssis porte-film; C, Face support du film; D, Vis de réglage; E, Cercle de Rowland.

Cette bride pleine reçoit une porte articulée (3G) contenant le châssis porte-film ( fig. 5).

Le réseau $(\varnothing=100 \mathrm{~mm}$; épaisseur $16 \mathrm{~mm}$ ) est contenu dans une boîte cylindrique (fig. 4 A). Deux tourillons (4 B) d'axe horizontal permettent l'orientation du boîtier.

Le support du boîtier comprend une première plate-forme (4 G) soudée à la cuve. Une seconde plateforme $(4 \mathrm{D})$ est posée sur la première et peut se déplacer suivant deux directions horizontales perpendiculaires, au moyen de quatre vis moletées réglables. Sur cette seconde plate-forme sont fixés trois supports : deux verticaux ( $4 \mathrm{E}$ ) reçoivent les tourillons du boîtier, le troisième $(4 \mathrm{~F})$ reçoit deux vis permettant de régler l'orientation du réseau.

Le châssis porte-film (5 B), maintenu sur un premier support (5 A) fixé au fond du couvercle, se déplace parallèlement aux raies du spectre, au moyen d'un dispositif à queue d'aronde. Ce déplacement, commandé de l'extérieur par l'intermédiaire d'un passage étanche, permet l'enregistrement de plusieurs spectres sur le même film, appliqué contre la face C. Des vis $(\mathrm{D})$ permettent en outre de légères rotations $\left( \pm 5^{\circ}\right)$ du châssis autour d'un axe horizontal pour faire coïncider parfaitement sa face cylindrique avec le cercle de Rowland.

La liaison entre la cuve du spectrographe et celle du plasmatron comporte la fente d'entrée $(3 \mathrm{~B})$, un cache en forme de $\mathrm{V}$ placé contre la fente (permettant de régler sa hauteur), un robinet à boisseau sphérique servant à la fois d'obturateur pour le rayonnement incident et de vanne d'isolement en pression des deux cuves où règnent des pressions différentes (environ $10^{-5}$ torr dans le spectrographe et de quelques torrs à 760 torrs dans l'enceinte du plasmatron).

Pour le choix des systèmes de pompage, il est nécessaire de tenir compte de trois faits essentiels :

1) Le rayonnement U.V. lointain est fortement absorbé par une grande majorité des gaz, et en particulier par l'oxygène moléculaire; il convient que la pression résiduelle de l'air dans le montage n'excède pas $10^{-4}$ torr;

2) Le débit de gaz rare (argon ou autre) introduit dans l'enceinte par le jet de plasma est important (environ $30 \mathrm{l} / \mathrm{mn}$ );

3) Les gaz s'écoulent à travers la fente du spectrographe durant les temps de pose.

Le vide est réalisé dans l'enceinte du spectrographe au moyen d'une pompe à palette Edwards 1SC $450 \mathrm{~B}$ et une pompe à diffusion d'huile Edwards $903 \mathrm{~F}$. Une vanne papillon Q.S.B. 9 et un piège à air liquide N.T.M. 9 A placés entre la pompe secondaire et l'enceinte empêchent les remontées de vapeurs d'huile et leur condensation sur le réseau.

\section{Source : le plasmatron en atmosphère raréfiée. -} Divers modèles de générateurs de plasma (arcs stabilisés ou chalumeaux) mis au point au laboratoire par Queffelec [1] et Velly [2] fonctionnent à la pression atmosphérique. Les études déjà faites par ces auteurs, ainsi que celles réalisées par Brossard et ses collabo- 
rateurs [13] sur la stabilité des générateurs de plasma en fonction du mode d'écoulement du gaz plasmagène, nous ont conduits à adopter un écoulement mixte (tourbillonnaire et longitudinal). Pour ce type d'écoulement et un débit de gaz donné, les proportions de gaz admises longitudinale et tangentielle peuvent

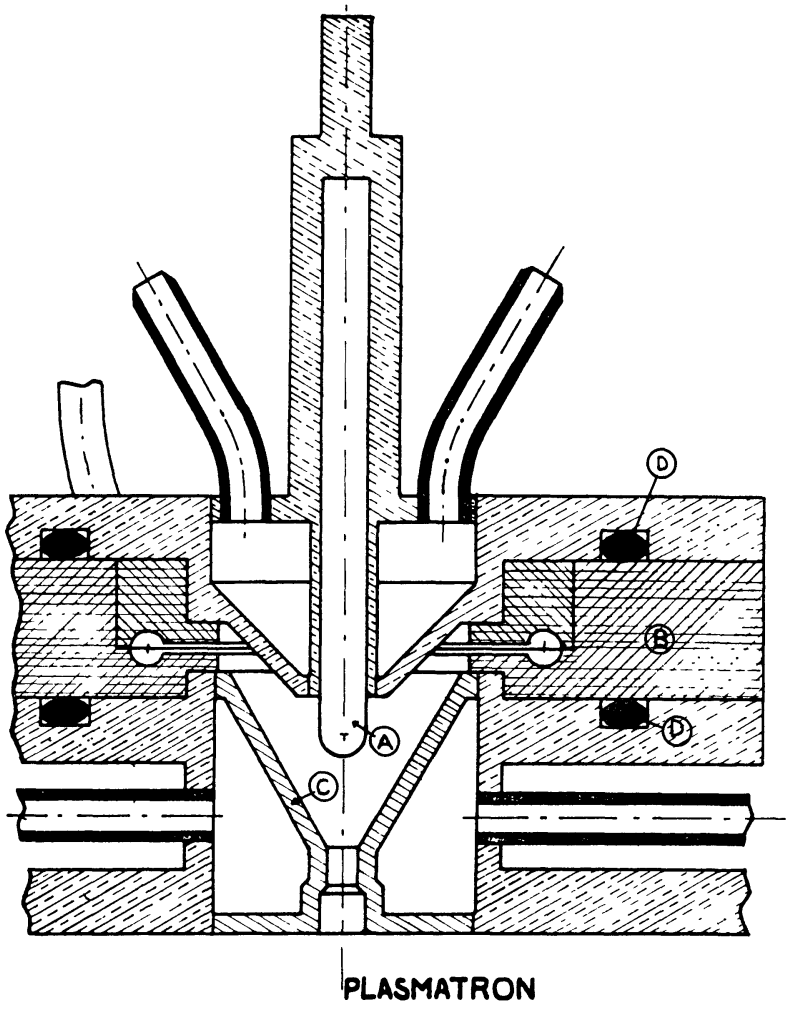

Fig. 6. - Plasmatron : A, Cathode en tungstène B, Isolant; C, Anode ; D, Joints d'étanchéité. varier dans de larges limites (25 à $75 \%$ environ du débit total) sans que la stabilité du jet soit altérée.

Pour des pressions dans l'enceinte inférieures à 1 atm, il est préférable, pour la stabilité de l'arc, d'utiliser une cathode dont l'extrémité est convexe.

Le plasmatron réalisé, de construction simple et d'une grande stabilité, se compose de trois parties essentielles ( fig. 6) :

a) La cathode (A) en tungstène thorié enfoncée dans un support en cuivre refroidi par une circulation d'eau;

b) L'anode $(\mathrm{C})$ en cuivre, refroidie par circulation d'eau, et de forme tronconique, prolongée par une buse, le diamètre du col étant de $4 \mathrm{~mm}$;

c) Deux pièces en afcodur (B) qui ont pour rôle, d'une part, d'isoler électriquement anode et cathode et, d'autre part, de contenir les arrivées de gaz dans la chambre de décharge. Le gaz arrive tangentiellement dans un tore par deux canalisations; une fente circulaire relie le tore à la cavité anodique. Ce dispositif permet de donner à la vitesse d'écoulement gazeux une composante longitudinale et une composante rotationnelle.

Deux joints toriques en perbunan (D) assurent l'étanchéité du plasmatron.

Une bride brasée sur l'anode permet de l'assujettir à l'enceinte à vide; celle-ci, cylindrique, se compose de trois tronçons et d'un couvercle à doubles parois renfermant un serpentin à circulation d'eau. Divers piquages permettent les raccords au spectrographe, à la pompe, aux manomètres, ainsi que l'observation directe du jet. Le vide (primaire) de cette cuve est obtenu au moyen d'une grosse pompe Edwards 1SG 3000 .

L'alimentation électrique du plasmatron est assurée

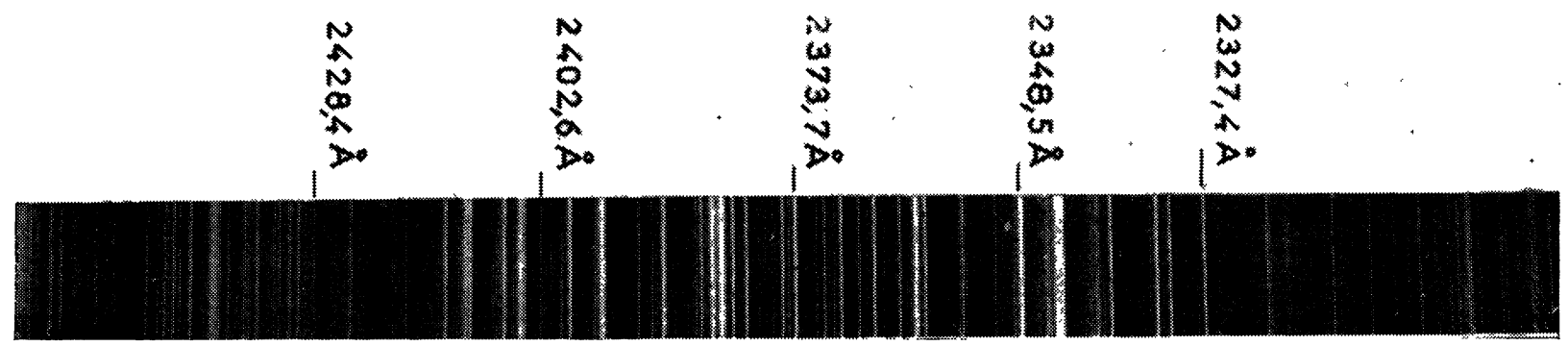

A

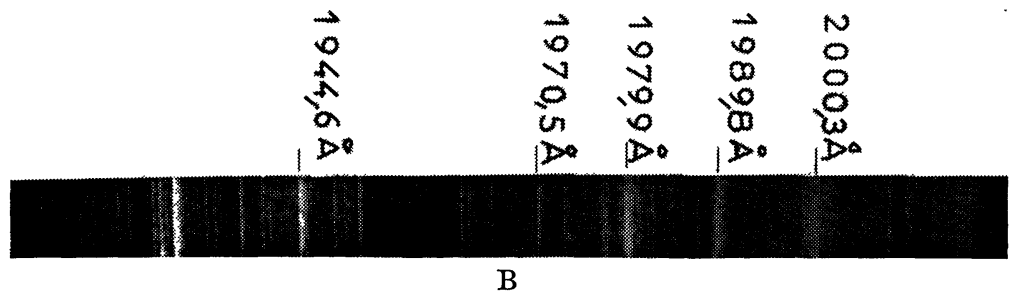

FIG. 7. - A, Spectre du fer; B, Spectre du cuivre dans un plasma d'argon. 
par un redresseur capable de fournir 200 A sous $300 \mathrm{~V}$ par redressement en double alternance des trois phases du secteur par l'intermédiaire d'un transformateur triphasé. En charge, le taux d'ondulation de la tension redressée est de 0,011 , ce qui nous donne un facteur de forme très voisin de 1 . Une résistance variable de $5 \Omega$, montée en série avec le plasmatron, permet de faire varier l'intensité du courant d'arc.

L'arc, amorcé par un igniteur, à $4 \mathrm{MHz}$, est d'une grande stabilité pour un débit gazeux de $30 \mathrm{l} / \mathrm{mn}$, un courant de $60 \mathrm{~A}$ et une pression variable à volonté, dans l'enceinte du plasmatron, de quelques torrs à $1 \mathrm{~atm}$. Une augmentation de la pression a pour effet de faire varier la longueur du dard.
5. Mise au point et étalonnage du spectrographe. - La possibilité d'observer sur un même spectre la tache centrale et les raies de longueurs d'onde de l'ordre de $2500 \AA$ a facilité les réglages préliminaires, complétés par contrôle photographique.

Un premier étalonnage des spectres de plasma a été effectué entre 2000 et $2500 \AA$ au moyen de l'arc au fer substitué au jet de plasma (fig. $7 \mathrm{~A}$ ).

Au-dessous de $2000 \AA$, nous avons utilisé les raies du cuivre qu'on peut faire apparaître dans le spectre du plasma dans certaines conditions de fonctionnement. Nous avons utilisé les longueurs d'onde du cuivre dans le vide, données par Shenstone [14] (fig. $7 \mathrm{~B})$.

A
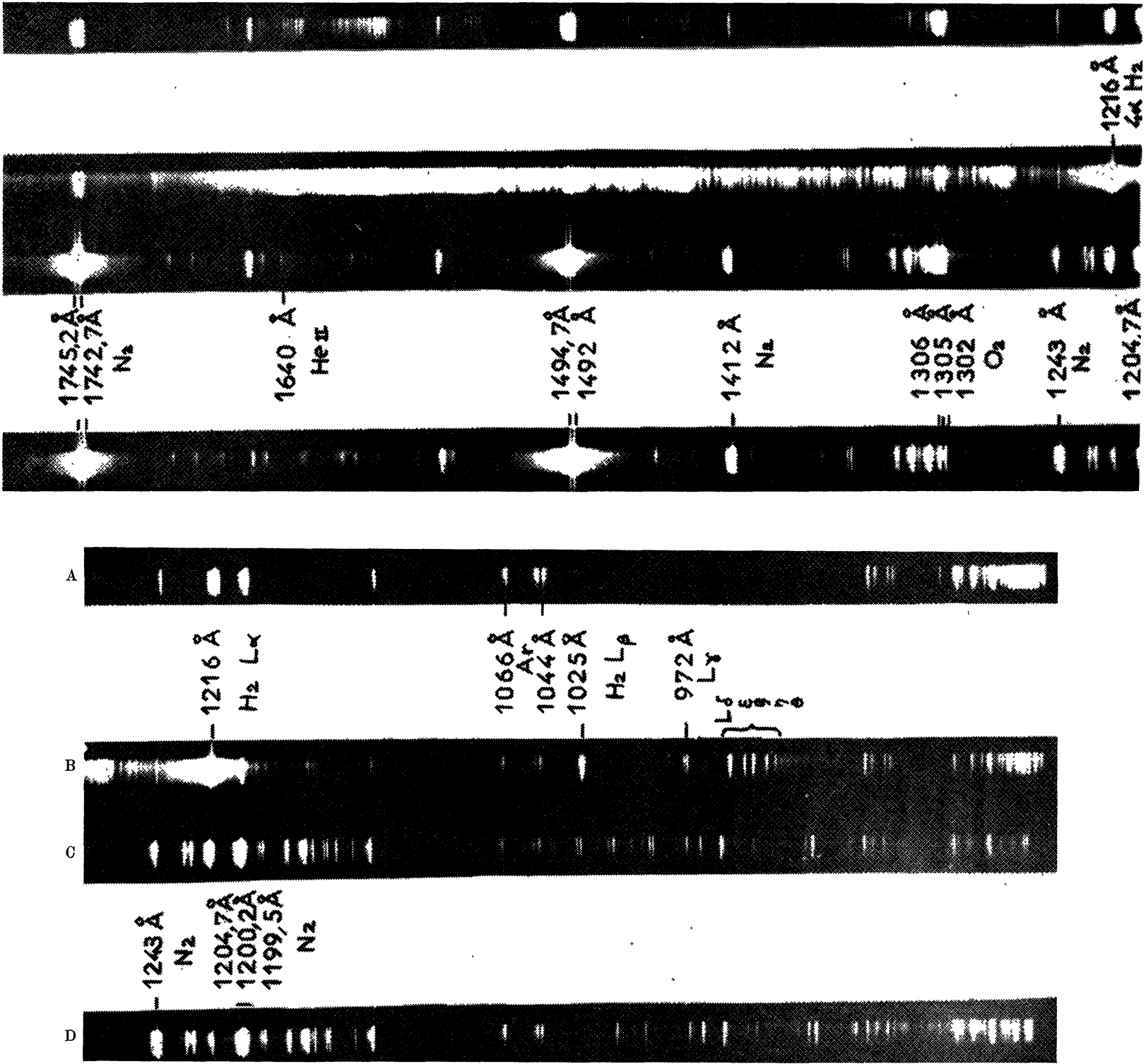

Fig. 8. - Spectre de l'argon : A, Argon pur; B, 3\% d'hydrogène; C, $3 \%$ d'hélium ; D, $3 \%$ d'azote. 
Vers les courtes longueurs d'onde, on repère sur les spectres du plasma des groupes de raies d'azote résiduel dans l'enceinte, notamment à $1745 \AA$ et $1494 \AA$.

Les spectres de mélanges gazeux (avec $I=60 \mathrm{~A}$ ) :

Argon $+3 \%$ d'hydrogène

Argon $+3 \%$ d'hélium

Argon $+3 \%$ d'azote

permettent de compléter l'étalonnage (fig. 8). Ces spectres font apparaître notamment la série de Lyman de l'hydrogène.

6. Conclusion. - Le spectrographe réalisé, de fonctionnement et de mise au point particulièrement simples, est principalement destiné à l'étude spectroscopique du dard d'un plasmatron de grande stabilité. Les spectres sont finement résolus.

L'arc du plasmatron utilise une intensité de courant variable de 40 à $80 \mathrm{~A}$.

Actuellement, nos travaux se poursuivent en vue de rendre le montage stigmatique et d'utiliser une source de plasma fonctionnant à $200 \mathrm{~A}$.

La dispersion de l'appareillage est suffisante pour nous permettre également l'étude dans l'U.V. lointain des formes de raies d'émission du gaz plasmagène à très haute température dans le plasmatron [15].

\section{BIBLIOGRAPHIE}

[1] QUEFFEL_ec (J.-L.), Thèse $3^{\mathrm{e}}$ cycle, Rennes, 1963.

[2] VELLY (J.-P.), Thèse $3^{\mathrm{e}}$ cycle, Rennes, 1966.

[3] LE MEN (R.), D.E.S., Physique, Rennes, 1967.

[4] Romand (J.) et VodAR (B.), Revue d'Optique, 1958, 37, 329.

[5] Romand (J.) et Vodar (B.), Revue d'Optique, 1960, 39, 167.

[6] Isaacs (L. D.), Price (W. C.) et Ridiefy (R. G.), Optica acta, 1960, 7, 263.

[7] DitchbuRN (R. W.), Optica acta, 1956, 3, 74-80.

[8] Milazzo (G.), Col. Spectr. Int. VI, 1956, 474-478.
[9] Burlitch (J. M.) et Duke (J. A.), Proc. West. Virgina Acad. Sc., 1959-60, 31-32, 71-73.

[10] SAWyeR (R. A.), "Experimental spectroscopy ", Dover Pub., New York, 1963.

[11] BEUTLER (H.), J.O.S.A., 1945, 35, 311.

[12] Griem (H. R.), "Plasma spectroscopy ", McGrawHill, New York, 1963.

[13] Brossard (J.), Denis (M.) et Fauchais (P.), Revue des hautes tempér. et réfract., 1964, nº 2.

[14] Shenstone (A. G.), Phil. Trans. Ray. Soc., 1936, A 751, 195.

[15] QUEFFELEC (J.-L.), à paraître. 